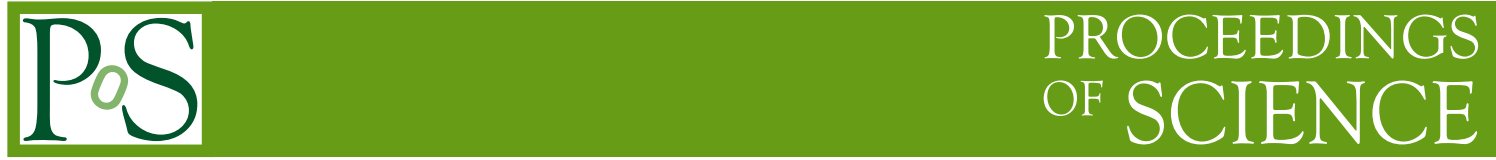

\title{
Contributions from flavour changing effective operators to the physics of the top quark at LHC
}

\section{Pedro Ferreira*}

CFTC da Universidade de Lisboa, Portugal

E-mail: ferreira@cii.fc.ul.pt

\section{R. Santos}

CFTC da Universidade de Lisboa, Portugal

E-mail: rsantos@cii.fc.ul.pt

We consider all relevant dimension six operators to perform a model independent analysis of flavour changing single top production at the LHC.

International Workshop on Top Quark Physics

January 12-15, 2006

Coimbra, Portugal

\footnotetext{
${ }^{*}$ Speaker.
} 


\section{Introduction}

The Large Hadron Collider (LHC) is starting its operation in 2007. In the low luminosity run, production of around 8 million top quark pairs per year can be anticipated. That is why the LHC is considered the ideal laboratory to study the heaviest of all known particles. Recently $[1,2]$ we undertook a model-independent study of possible new physics effects on the phenomenology of the top quark. Following reference [3] we considered a set of dimension six effective operators and analyzed its impact on observable quantities related to the top quark, such as its width or the cross section for single top quark production at the LHC. Due to the large number of arbitrary coupling constants, we have excluded the ones with little or no impact on phenomena occurring at energy scales inferior to the LHC's. This framework of effective lagrangians has been widely used to study the top particle $[4,5,6,7,8,9]$.

Let us remark that our philosophy in $[1,2]$ was also somewhat different from that of most previous works in this field, in that we presented, whenever possible, analytical expressions. Our aim was, and is, to provide our experimental colleagues with formulae they can use directly in their Monte Carlo simulations.

\section{Effective operator formalism}

The effective operator approach is based on the assumption that, at a given energy scale $\Lambda$, physics effects beyond those predicted by the SM make themselves manifest. We describe this by assuming the lagrangean

$$
\mathscr{L}=\mathscr{L}^{S M}+\frac{1}{\Lambda} \mathscr{L}^{(5)}+\frac{1}{\Lambda^{2}} \mathscr{L}^{(6)}+O\left(\frac{1}{\Lambda^{3}}\right)
$$

where $\mathscr{L}^{S M}$ is the SM lagrangean and $\mathscr{L}^{(5)}$ and $\mathscr{L}^{(6)}$ are all of the dimension 5 and 6 operators which, like $\mathscr{L}^{S M}$, are invariant under the gauge symmetries of the SM. The $\mathscr{L}^{(5)}$ terms break baryon and lepton number conservation, and are thus not usually considered. This leaves us with the $\mathscr{L}^{(6)}$ operators, some of which, after spontaneous symmetry breaking, generate dimension five terms. The list of dimension six operators is quite vast [3], therefore some sensible criteria of selection are needed. Underlying all our work is the desire to study a new possible type of physics, flavour changing strong interactions. The first criterion is to choose those $\mathscr{L}^{(6)}$ operators that have no sizeable impact on low energy physics (below the $\mathrm{TeV}$ scale, say). Another criterion was to only consider operators with a single top quark, since we will limit our studies to processes of single top production. Finally, we will restrict ourselves to operators with gluons, or four-fermion ones. No effective operators with electroweak gauge bosons will be considered.

The gluon operators that survive these criteria are but two, which, in the notation of ref. [3], are written as

$$
\begin{aligned}
\mathscr{O}_{u G} & =i \frac{\alpha_{i j}}{\Lambda^{2}}\left(\bar{u}_{R}^{i} \lambda^{a} \gamma^{\mu} D^{v} u_{R}^{j}\right) G_{\mu \nu}^{a} \\
\mathscr{O}_{u G \phi} & =\frac{\beta_{i j}}{\Lambda^{2}}\left(\bar{q}_{L}^{i} \lambda^{a} \sigma^{\mu v} u_{R}^{j}\right) \tilde{\phi} G_{\mu \nu}^{a} .
\end{aligned}
$$


$q_{L}$ and $u_{R}$ are spinors (a left quark doublet and up-quark right singlet of $S U(2)$, respectively), $\tilde{\phi}$ is the charge conjugate of the Higgs doublet and $G_{\mu \nu}^{a}$ is the gluon tensor. $\alpha_{i j}$ and $\beta_{i j}$ are complex dimensionless couplings, the $(i, j)$ being flavour indices. According to our criteria, one of these indices must belong to the third generation. After spontaneous symmetry breaking the neutral component of the field $\phi$ acquires a vev $\left(\phi_{0} \rightarrow \phi_{0}+v\right.$, with $\left.v=246 / \sqrt{2} \mathrm{GeV}\right)$ and the second of these operators generates a dimension five term. The lagrangean for new physics thus becomes

$$
\begin{aligned}
\mathscr{L}= & \alpha_{t u} \mathscr{O}_{t u}+\alpha_{u t} \mathscr{O}_{u t}+\beta_{t u} \mathscr{O}_{t u \phi}+\beta_{u t} \mathscr{O}_{u t \phi}+\text { h.c. } \\
= & \frac{i}{\Lambda^{2}}\left[\alpha_{t u}\left(\bar{t}_{R} \lambda^{a} \gamma^{\mu} D^{v} u_{R}\right)+\alpha_{u t}\left(\bar{u}_{R} \lambda^{a} \gamma^{\mu} D^{v} t_{R}\right)\right] G_{\mu v}^{a}+ \\
& \frac{v}{\Lambda^{2}}\left[\beta_{t u}\left(\bar{t}_{L} \lambda^{a} \sigma^{\mu v} u_{R}\right)+\beta_{u t}\left(\bar{u}_{L} \lambda^{a} \sigma^{\mu v} t_{R}\right)\right] G_{\mu v}^{a}+\text { h.c. } .
\end{aligned}
$$

Several extensions of the SM, such as supersymmetry and two Higgs doublet models, may generate contributions to this type of operator [10]. The Feynman rules for these anomalous vertices are shown in figure (1), with quark momenta following the arrows and incoming gluon momenta.
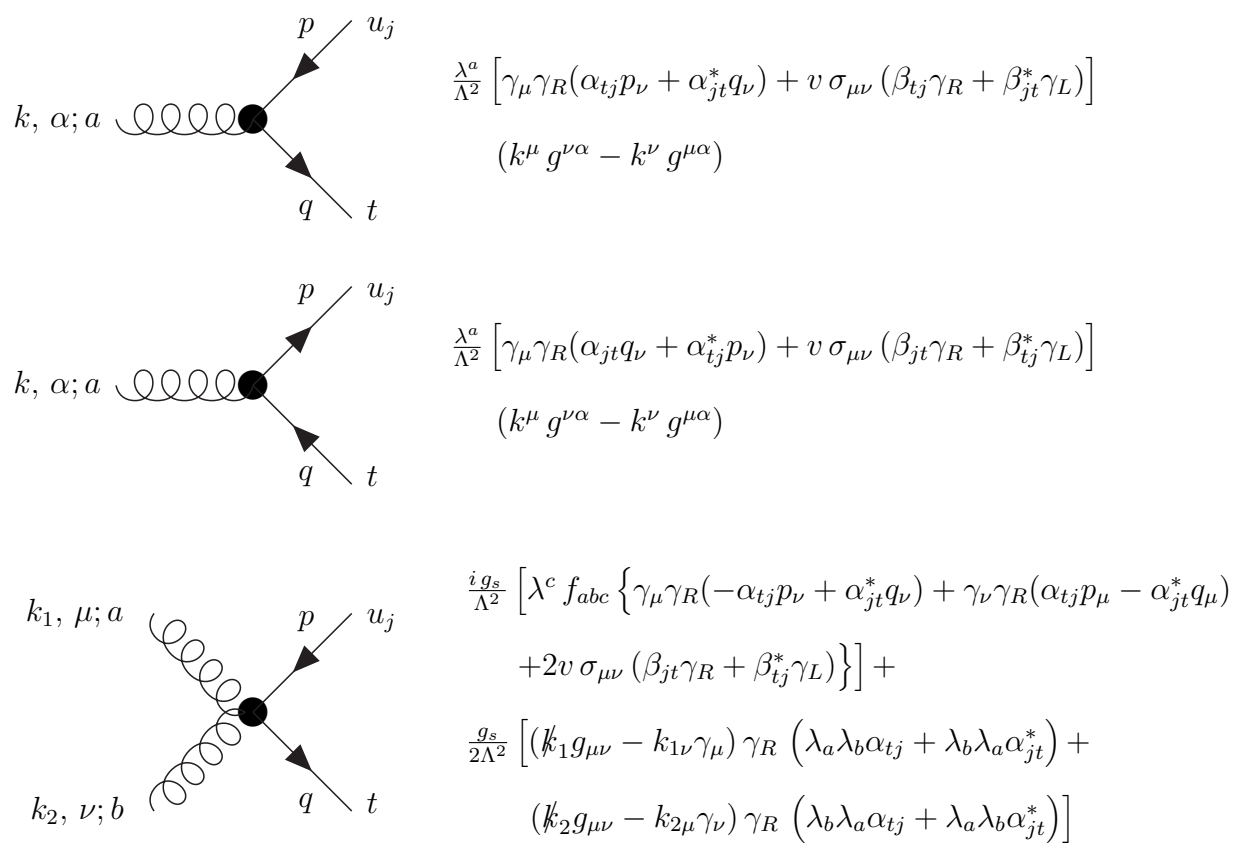

Figure 1: Feynman rules for anomalous gluon vertices.

In ref. [1] we calculated the effect of these operators on the width of the quark top. They allow for the decay $t \rightarrow u g(t \rightarrow c g$ ) (which is also possible in the SM, albeit at higher orders), and the corresponding width is given by

$$
\Gamma(t \rightarrow u g)=\frac{m_{t}^{3}}{12 \pi \Lambda^{4}}\left\{m_{t}^{2}\left|\alpha_{u t}+\alpha_{t u}^{*}\right|^{2}+16 v^{2}\left(\left|\beta_{t u}\right|^{2}+\left|\beta_{u t}\right|^{2}\right)+8 v m_{t} \operatorname{Im}\left[\left(\alpha_{u t}+\alpha_{t u}^{*}\right) \beta_{t u}\right]\right\}
$$


and an analogous expression for $\Gamma(t \rightarrow c g)$. In this expression, and throughout the entire paper, we will consider all quark masses, except the top's, equal to zero; the imprecision introduced by this approximation is extremely small, as we verified having performed the full calculations. Direct top production is also possible with these new vertices (meaning, the production of a top quark from partonic reactions such as $g u \rightarrow t$ or $g c \rightarrow t$ ), and the corresponding cross section at the LHC is given by

$$
\sigma(p p \rightarrow t)=\sum_{q=u, c} \Gamma(t \rightarrow q g) \frac{\pi^{2}}{m_{t}^{2}} \int_{m_{t}^{2} / E_{C M}^{2}}^{1} \frac{2 m_{t}}{E_{C M}^{2} x_{1}} f_{g}\left(x_{1}\right) f_{q}\left(m_{t}^{2} /\left(E_{C M}^{2} x_{1}\right)\right) d x_{1}
$$

In this expression $E_{C M}$ is the proton-proton center-of-mass energy (14 $\mathrm{TeV}$ at the $\mathrm{LHC}$ ) and $f_{g}$ and $f_{q}$ are the parton density functions of the gluon and quark, respectively.

Notice how both the top width (2.4) and the cross section (2.5) depend on $\Lambda^{-4}$. There are processes with a $\Lambda^{-2}$ dependence, namely the interference terms between the anomalous operators and the SM diagrams of single top quark production, via the exchange of a $\mathrm{W}$ gauge boson processes like $u \bar{d} \rightarrow t \bar{d}$. They were studied in ref. [1] in detail, and we discovered that, due to a strong CKM suppression, the contributions from the anomalous vertices are extremely small.

Now, the operators that compose the lagrangean (2.3) are not, in fact, completely independent. If one performs integrations by parts and uses the fermionic equations of motion [3, 11], one obtains the following relations between them:

$$
\begin{aligned}
& \mathscr{O}_{u t}^{\dagger}=\mathscr{O}_{t u}-\frac{i}{2}\left(\Gamma_{u}^{\dagger} \mathscr{O}_{u t \phi}^{\dagger}+\Gamma_{u} \mathscr{O}_{t u \phi}\right) \\
& \mathscr{O}_{u t}^{\dagger}=\mathscr{O}_{t u}-i g_{s} \bar{t} \gamma_{\mu} \gamma_{R} \lambda^{a} u \sum_{i}\left(\bar{u}^{i} \gamma^{\mu} \gamma_{R} \lambda_{a} u^{i}+\bar{d}^{i} \gamma^{\mu} \gamma_{R} \lambda_{a} d^{i}\right)
\end{aligned}
$$

where $\Gamma_{u}$ are the Yukawa couplings of the up quark and $g_{s}$ the strong coupling constant. In the second of these equations we see the appearance of four-fermion terms, indicating that they have to be taken into account in these studies. Equations (2.6) then tell us that there are two relations between the several operators, which means that we are allowed to set two of the couplings to zero.

A careful analysis of the operators listed in [3] leads us to consider three types of four-fermion operators:

- Type 1 ,

$$
\mathscr{O}_{u_{1}}=\frac{g_{s} \gamma_{u_{1}}}{\Lambda^{2}}\left(\bar{t} \lambda^{a} \gamma^{\mu} \gamma_{R} u\right)\left(\bar{q} \lambda^{a} \gamma_{\mu} \gamma_{R} q\right)+\text { h.c. }
$$

where $q$ is any given quark, other than the top;

- Type 2 ,

$$
\mathscr{O}_{u_{2}}=\frac{g_{s} \gamma_{u_{2}}}{\Lambda^{2}}\left[\left(\bar{t} \lambda^{a} \gamma_{L} u^{\prime}\right)\left(\bar{u}^{\prime \prime} \lambda^{a} \gamma_{R} u\right)+\left(\bar{t} \lambda^{a} \gamma_{L} d^{\prime}\right)\left(\bar{d}^{\prime \prime} \lambda^{a} \gamma_{R} u\right)\right]+\text { h.c. },
$$

with down and up quarks from several possible generations, excluding the top once more;

- Type 3,

$$
\mathscr{O}_{u_{3}}=\frac{g_{s} \gamma_{u_{3}}}{\Lambda^{2}}\left[\left(\bar{t} \lambda^{a} \gamma_{R} u\right)\left(\bar{b} \lambda^{a} \gamma_{R} d^{\prime}\right)-\left(\bar{t} \lambda^{a} \gamma_{R} d^{\prime}\right)\left(\bar{b} \lambda^{a} \gamma_{R} u\right)\right]+\text { h.c. }
$$


and also,

$$
\frac{g_{s} \gamma_{u_{3}}^{*}}{\Lambda^{2}}\left[\left(\bar{t} \lambda^{a} \gamma_{L} u\right)\left(\bar{d}^{\prime} \lambda^{a} \gamma_{L} d^{\prime \prime}\right)-\left(\bar{t} \lambda^{a} \gamma_{L} d\right)\left(\bar{d}^{\prime} \lambda^{a} \gamma_{L} u^{\prime \prime}\right)\right]+\text { h.c. } .
$$

The $\gamma_{u}$ 's are complex couplings. We of course consider identical operators for the case of flavour changing interactions with the $c$ quark. In the notation of ref. [3] these operators correspond, respectively, to $\bar{R} R \bar{R} R, \bar{L} R \bar{R} L$ and $\bar{L} R \widetilde{(\bar{L} R)}$, in the octet configuration. We could have also considered the singlet operators but, since their spinorial structure is identical to these (lacking only the GellMann matrices) we opted to leave them out. The presence of the $\lambda^{a}$ in these operators also signals their origin within the strong interaction sector, in line with our aim of studying strong flavour changing effects. For this reason, and for an easier comparison between the effects of the several operators, we included, in the definitions of the four-fermion terms above, an overall factor of $g_{s}$.

\section{Cross sections for $g g \rightarrow t \bar{u}$ and $g u \rightarrow g t$. Four-fermion channels.}

The Feynman diagrams contributing to the partonic cross sections, $g g \rightarrow t \bar{u}$ and $g u \rightarrow g t$ are shown in figs. (2) and (3) respectively. Details of the calculations can be found in [2].
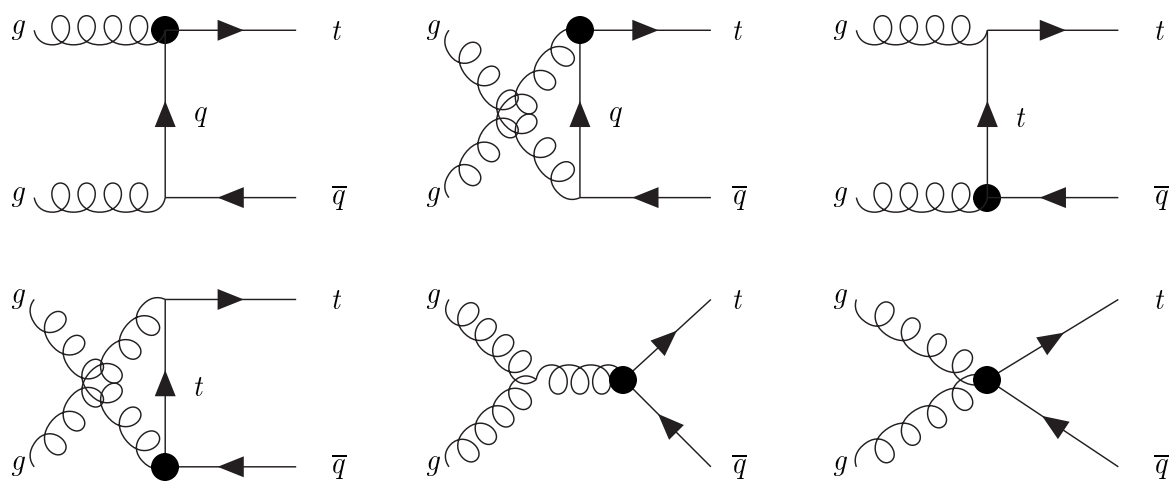

Figure 2: Feynman diagrams for the two-gluon channel.

If we assume that the branching ratio $B R(t \rightarrow b W)$ is approximately $100 \%$ and use $\Gamma(t \rightarrow$ $b W)=1.42\left|V_{t b}\right|^{2} \mathrm{GeV}$ (a value which includes QCD corrections) [13], we may express the partial widths as $\Gamma(t \rightarrow q g)=1.42\left|V_{t b}\right|^{2} B R(t \rightarrow q g)$. In terms of these branching ratios, and using the CTEQ6M structure functions [14] ${ }^{1}$ to perform the integration in the pdf's, we obtain, for the total cross sections, the following results (expressed in picobarn):

$$
\begin{aligned}
& \sigma(p p \rightarrow g g \rightarrow t \bar{q})=[0.5 B R(t \rightarrow u g)+0.5 B R(t \rightarrow c g)]\left|V_{t b}\right|^{2} 10^{4} \\
& \sigma(p p \rightarrow g g \rightarrow \bar{t} q)=\sigma(p p \rightarrow g g \rightarrow t \bar{q}) \\
& \sigma(p p \rightarrow g q \rightarrow g t)=[8.2 B R(t \rightarrow u g)+0.8 B R(t \rightarrow c g)]\left|V_{t b}\right|^{2} 10^{4} \\
& \sigma(p p \rightarrow g \bar{q} \rightarrow g \bar{t})=[1.5 B R(t \rightarrow u g)+0.8 B R(t \rightarrow c g)]\left|V_{t b}\right|^{2} 10^{4}
\end{aligned}
$$

\footnotetext{
${ }^{1}$ We used a factorization scale equal to the mass of the quark top, that being the characteristic scale of these reactions. This choice of $\mu_{F}$ produces smaller cross section values than, saying, choosing it equal to the partonic center-of-mass energy [15].
} 

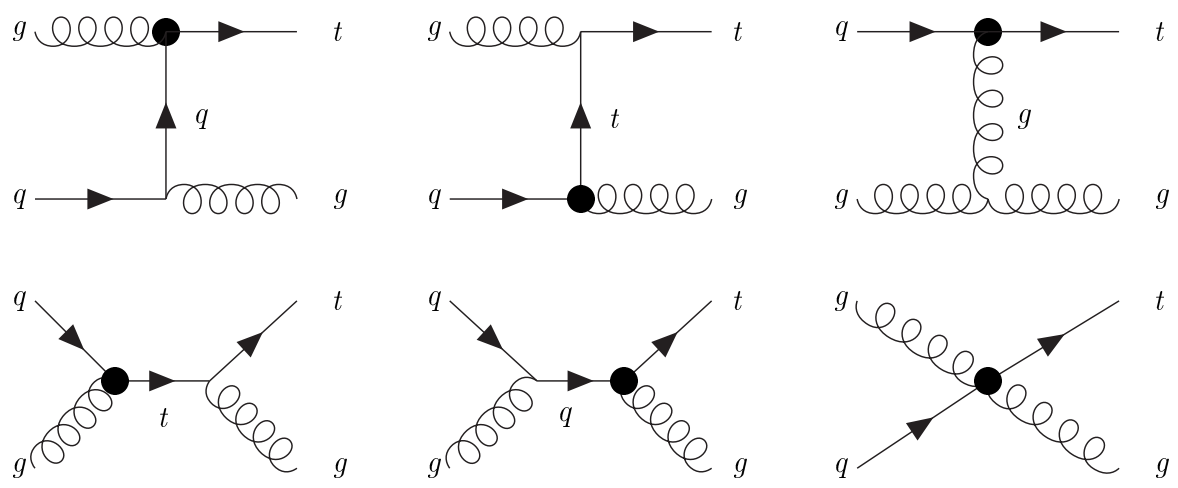

Figure 3: Feynman diagrams for the gluon-quark channel.

and for the direct top cross section we have,

$$
\begin{aligned}
& \sigma(p p \rightarrow g q \rightarrow t)=[10.5 B R(t \rightarrow u g)+1.6 B R(t \rightarrow c g)]\left|V_{t b}\right|^{2} 10^{4} \\
& \sigma(p p \rightarrow g \bar{q} \rightarrow \bar{t})=[2.7 B R(t \rightarrow u g)+1.6 B R(t \rightarrow c g)]\left|V_{t b}\right|^{2} 10^{4} .
\end{aligned}
$$

The larger values of the coefficients affecting the up-quark branching ratios in eqs. (3.1) and (3.2) derive from the fact that the pdf for that quark is larger than the charm's. The numerical integration has an error of less than one percent. Except for the direct top channel, all of these cross sections (as well as the four-fermion results we will soon present) are integrated with a cut on the transverse momentum $\left(p_{T}\right)$ of the light parton in the final state of $15 \mathrm{GeV}$. This is to remove the collinear and soft singularities in the gluon-quark subprocesses to render finite partonic cross sections, for a finite $p_{T}$ cut eliminates both of those divergences in two-to-two scattering processes. In a realistic analysis including backgrounds, a higher $p_{T}$ cut might well be needed, to suppress background rates in order to observe the signal events. That study, however, is beyond the scope of this work. Observe how the direct channel cross section is larger than the others. Notice, however, that due to the kinematics of that channel, no $p_{T}$ cut was applied. When imposing such a cut on the decay products of the top quark produced in the direct channel, the corresponding cross section will certainly be reduced.

It is quite remarkable that these cross sections are all proportional to the branching ratios for rare decays of the top. These are possible even within the SM, at higher orders. For instance, one expects the SM value of $B R(t \rightarrow c g)$ to be of about $10^{-12}[10,16], B R(t \rightarrow u g)$ two orders of magnitude smaller. What this means is that, if whatever new physics lies beyond the SM has no sizeable impact on the flavour changing decays of the top quark, so that its branching ratios are not substantially different from their SM values, then one does not expect any excess of single top production at the LHC through these channels. On the other hand, if an excess of single top production is observed, even a small one, the expressions (3.1) and (3.2) tell us that $B R(t \rightarrow c g)$ and $B R(t \rightarrow u g)$ will have to be very different from their SM values. In fact, in models with two Higgs doublets or supersymmetry, one expects the branching ratios $B R(t \rightarrow c g)$ and $B R(t \rightarrow u g)$ to increase immensely $[10,16]$, in some models becoming as large as $\sim 10^{-4}$. If that is the case, 
eqs. (3.1) and (3.2) predict a significant increase in the cross section for single top production at the LHC. This cross section is therefore a very sensitive observable to probe for new physics.

A single top in the final state can also be produced through quark-quark or quark-antiquark scattering. The complete list of processes is $u u \rightarrow t u, u c \rightarrow t c, u \bar{u} \rightarrow t \bar{u}, u \bar{u} \rightarrow t \bar{c}, u \bar{c} \rightarrow t \bar{c}$, $d \bar{d} \rightarrow t \bar{u}, u d \rightarrow t d$ and $u \bar{d} \rightarrow t \bar{d}$. We have however excluded from this list, processes that are not consistent with our choice of gluonic operators, like, for instance, $s \bar{d} \rightarrow t \bar{u}$. In fig. (4) we show the Feynman diagrams for the process $u u \rightarrow t u$ and the details of the calculation can again be found in [2].
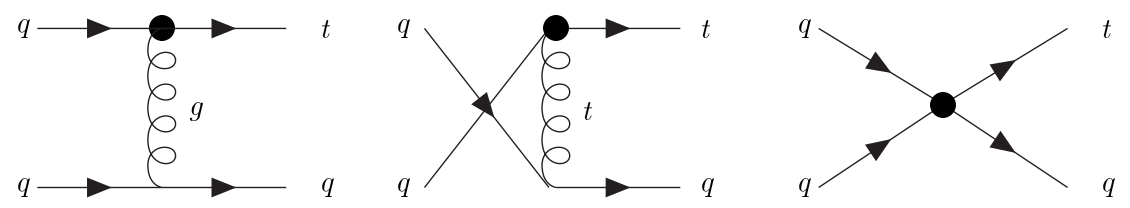

Figure 4: Feynman diagrams for $q q \rightarrow q t$. The four-fermion graph can generate both " $t$-channel" and " $u$-channel" contributions.

\section{Results and discussion}

We can now gather all the results obtained in refs. [1,2] for the cross sections of single top production. In terms of the couplings, the direct channel, eq. (3.2), gives us

$$
\sigma_{g u \rightarrow t}=\left\{321\left|\alpha_{u t}+\alpha_{t u}^{*}\right|^{2}+5080\left(\left|\beta_{t u}\right|^{2}+\left|\beta_{u t}\right|^{2}\right)+2556 \operatorname{Im}\left[\left(\alpha_{u t}+\alpha_{t u}^{*}\right) \beta_{t u}\right]\right\} \frac{1}{\Lambda^{4}} \mathrm{pb},
$$

for the partonic channel $g u \rightarrow t$. For the gluon-gluon and gluon-quark channels, we have, from eqs. (3.1),

$$
\begin{aligned}
& \sigma_{g g \rightarrow t \bar{u}}=\left\{14\left|\alpha_{u t}+\alpha_{t u}^{*}\right|^{2}+221\left(\left|\beta_{t u}\right|^{2}+\left|\beta_{u t}\right|^{2}\right)+111 \operatorname{Im}\left[\left(\alpha_{u t}+\alpha_{t u}^{*}\right) \beta_{t u}\right]\right\} \frac{1}{\Lambda^{4}} \mathrm{pb} \\
& \sigma_{g u \rightarrow g t}=\left\{250\left|\alpha_{u t}+\alpha_{t u}^{*}\right|^{2}+3952\left(\left|\beta_{t u}\right|^{2}+\left|\beta_{u t}\right|^{2}\right)+1988 \operatorname{Im}\left[\left(\alpha_{u t}+\alpha_{t u}^{*}\right) \beta_{t u}\right]\right\} \frac{1}{\Lambda^{4}} \mathrm{pb} .
\end{aligned}
$$

Finally, the four-fermion processes can all be gathered (after integration on the parton density functions, as before) in a single expression,

$$
\begin{aligned}
\sigma_{4 F}^{(u)}=[ & 171\left|\alpha_{u t}\right|^{2}+179\left|\alpha_{t u}\right|^{2}-176 \operatorname{Re}\left(\alpha_{u t} \alpha_{t u}\right)+331 \operatorname{Im}\left(\alpha_{u t} \beta_{t u}\right)-362 \operatorname{Im}\left(\alpha_{t u} \beta_{t u}^{*}\right) \\
& +689\left(\left|\beta_{t u}\right|^{2}+\left|\beta_{u t}\right|^{2}\right)+177 \operatorname{Re}\left(\alpha_{u t} \gamma_{u_{1}}\right)-185 \operatorname{Re}\left(\alpha_{t u} \gamma_{u_{1}}^{*}\right)-16 \operatorname{Im}\left(\beta_{t u} \gamma_{u_{1}}^{*}\right) \\
& -17 \operatorname{Re}\left(\alpha_{u t} \gamma_{u_{2}}\right)+17 \operatorname{Re}\left(\alpha_{t u} \gamma_{u_{2}}^{*}\right)+0.1 \operatorname{Im}\left(\beta_{t u} \gamma_{u_{2}}^{*}\right) \\
& \left.+525\left|\gamma_{u_{1}}\right|^{2}+94\left|\gamma_{u_{2}}\right|^{2}+88\left|\gamma_{u_{3}}\right|^{2}\right] \frac{1}{\Lambda^{4}} \mathrm{pb} .
\end{aligned}
$$

For the channels proceeding through the charm quark, we have analogous expressions, with different numeric values in most cases due to different parton content inside the proton. Within the 
four-fermion cross sections we show the results for the production of a bottom quark alongside the top, through the processes $u b \rightarrow t b$ and $u \bar{b} \rightarrow t \bar{b}$ (and analogous processes for the $c$ quark). They are given by

$$
\begin{aligned}
& \sigma_{t+b}^{(u)}=\left[8\left|\alpha_{u t}\right|^{2}+9\left|\alpha_{t u}\right|^{2}-2 \operatorname{Re}\left(\alpha_{u t} \alpha_{t u}\right)+28 \operatorname{Im}\left(\alpha_{u t} \beta_{t u}\right)-32 \operatorname{Im}\left(\alpha_{t u} \beta_{t u}^{*}\right)\right. \\
& +59\left(\left|\beta_{t u}\right|^{2}+\left|\beta_{u t}\right|^{2}\right)+12 \operatorname{Re}\left(\alpha_{u t} \gamma_{u_{1}}\right)-13 \operatorname{Re}\left(\alpha_{t u} \gamma_{u_{1}}^{*}\right)-3 \operatorname{Im}\left(\beta_{t u} \gamma_{u_{1}}^{*}\right) \\
& -2 \operatorname{Re}\left(\alpha_{u t} \gamma_{u_{2}}\right)+2 \operatorname{Re}\left(\alpha_{t u} \gamma_{u_{2}}^{*}\right)+0.5 \operatorname{Im}\left(\beta_{t u} \gamma_{u_{2}}^{*}\right) \\
& \left.+19\left|\gamma_{u_{1}}\right|^{2}+5\left|\gamma_{u_{2}}\right|^{2}+16\left|\gamma_{u_{3}}\right|^{2}\right] \frac{1}{\Lambda^{4}} \mathrm{pb} \\
& \text { and } \quad \sigma_{t+b}^{(c)}=\left[0.4\left|\alpha_{c t}\right|^{2}+0.6\left|\alpha_{t c}\right|^{2}+0.2 \operatorname{Re}\left(\alpha_{c t} \alpha_{t c}\right)+2 \operatorname{Im}\left(\alpha_{c t} \beta_{t c}\right)-3 \operatorname{Im}\left(\alpha_{t c} \beta_{t c}^{*}\right)\right. \\
& \left.+5\left(\left|\beta_{t c}\right|^{2}+\left|\beta_{c t}\right|^{2}\right)+\left|\gamma_{c_{1}}\right|^{2}+0.2\left|\gamma_{c_{2}}\right|^{2}+0.6\left|\gamma_{c_{3}}\right|^{2}\right] \frac{1}{\Lambda^{4}} \mathrm{pb}
\end{aligned}
$$

where the interference terms between the $\{\alpha, \beta\}$ and the $\gamma$ were left out because they were too small when compared with the remaining terms.

Finally, by changing the pdf integrations, we can also obtain the cross sections for anti-top production.

We have thus far presented the complete expressions for the cross sections but, as was discussed earlier and is made manifest by equation (2.6), some of the operators we considered are not independent. In fact, eq. (2.6) implies that we can choose two of the couplings $\left\{\alpha_{u t}, \alpha_{t u}, \beta_{u t}, \beta_{t u}, \gamma_{u_{1}}\right\}$ to be equal to zero. Notice that $\gamma_{u_{2}}$ and $\gamma_{u_{3}}$ are not included in this choice, as the respective operators do not enter into equations (2.6). A similar conclusion may be drawn, of course, about the couplings $\left\{\alpha_{c t}, \alpha_{t c}, \beta_{c t}, \beta_{t c}, \gamma_{c_{1}}\right\}$. We choose to set $\beta_{t u}$ and $\gamma_{u_{1}}$ to zero, as this choice eliminates many of the interference terms of the cross sections. Summing all of the different contributions, we obtain, for the single top production cross section, the following results:

$$
\begin{aligned}
\sigma_{\text {single t }}^{(u)}= & {\left[756\left|\alpha_{u t}\right|^{2}+764\left|\alpha_{t u}\right|^{2}+994 \operatorname{Re}\left(\alpha_{u t} \alpha_{t u}\right)+9942\left|\beta_{u t}\right|^{2}\right.} \\
& \left.-17 \operatorname{Re}\left(\alpha_{u t} \gamma_{u_{2}}\right)+17 \operatorname{Re}\left(\alpha_{t u} \gamma_{u_{2}}^{*}\right)+94\left|\gamma_{u_{2}}\right|^{2}+88\left|\gamma_{u_{3}}\right|^{2}\right] \frac{1}{\Lambda^{4}} \mathrm{pb} \\
\sigma_{\text {single } t}^{(c)}= & {\left[109\left|\alpha_{c t}\right|^{2}+109\left|\alpha_{t c}\right|^{2}+166 \operatorname{Re}\left(\alpha_{c t} \alpha_{t c}\right)+1514\left|\beta_{c t}\right|^{2}\right.} \\
& \left.-3 \operatorname{Re}\left(\alpha_{c t} \gamma_{c_{2}}\right)+3 \operatorname{Re}\left(\alpha_{t c} \gamma_{c_{2}}^{*}\right)+24\left|\gamma_{c_{2}}\right|^{2}+27\left|\gamma_{c_{3}}\right|^{2}\right] \frac{1}{\Lambda^{4}} \mathrm{pb}
\end{aligned}
$$

For anti-top production,

$$
\begin{aligned}
\sigma_{\text {single } \bar{t}}^{(u)}= & {\left[174\left|\alpha_{u t}\right|^{2}+174\left|\alpha_{t u}\right|^{2}+265 \operatorname{Re}\left(\alpha_{u t} \alpha_{t u}\right)+2422\left|\beta_{u t}\right|^{2}\right.} \\
& \left.-\operatorname{Re}\left(\alpha_{u t} \gamma_{u_{2}}\right)+3 \operatorname{Re}\left(\alpha_{t u} \gamma_{u_{2}}^{*}\right)+26\left|\gamma_{u_{2}}\right|^{2}+35\left|\gamma_{u_{3}}\right|^{2}\right] \frac{1}{\Lambda^{4}} \mathrm{pb} \\
\sigma_{\text {single } \bar{t}=}^{(c)}= & 109\left|\alpha_{c t}\right|^{2}+109\left|\alpha_{t c}\right|^{2}+166 \operatorname{Re}\left(\alpha_{c t} \alpha_{t c}\right)+1514\left|\beta_{c t}\right|^{2} \\
& \left.-7 \operatorname{Re}\left(\alpha_{c t} \gamma_{c_{2}}\right)+7 \operatorname{Re}\left(\alpha_{t c} \gamma_{c_{2}}^{*}\right)+29\left|\gamma_{c_{2}}\right|^{2}+29\left|\gamma_{c_{3}}\right|^{2}\right] \frac{1}{\Lambda^{4}} \mathrm{pb}
\end{aligned}
$$

There is an extensive literature on the subject of single top production [17]. For the LHC, the $\mathrm{SM}$ prediction is usually considered to be $319.7 \pm 19.3 \mathrm{pb}$ [15]. Considering the large numbers we 
are obtaining in the expressions above - specially the coefficients of the $\beta$ couplings, though the others are not in any way negligible - we can see that even a small deviation from the SM framework will produce a potentially large effect in this cross section. It is indeed a good observable to test new physics, as it seems so sensible to its presence. Alternatively, if the cross section for single top production at the LHC is measured in the years to come and is found to be in complete agreement with the SM predicted value, then we will be able to set extremely stringent bounds on the couplings $\{\alpha, \beta, \gamma\}$ - on new physics in general - precisely for the same reasons.

In conclusion, we have calculated the contributions from a large set of dimension six operators to cross sections of several processes of single top production at the LHC. All cross sections involving gluons in the initial or final states are proportional to branching ratios of rare top quark decays. This makes these processes extremely sensitive to new physics, since those branching ratios may vary by as much as eight orders of magnitude in the SM and extended models. The four-fermion operators we chose break this proportionality so that, even if the branching ratios of the top quark conform to those of the SM, we may still have an excess of single top production at the LHC, stemming from those same operators. One of the advantages of working in a fully gauge-invariant manner is the possibility of using the equations of motion to introduce relations between the operators and thus reduce the number of independent parameters. One possible further simplification, if one so wishes, would be to consider each generation's couplings related by the SM CKM matrix elements, so that, for instance, $\alpha_{t u}=\alpha_{t c}\left|V_{u b} / V_{c b}\right|$. This should constitute a reasonable estimate of the difference in magnitude between each generations' couplings. Finally, in this paper we presented both the total anomalous cross sections for single top production and those of the individual processes that contribute to it. If there is any experimental method - through kinematical cuts or jet analysis - to distinguish between each of the possible partonic channels (direct top production; gluon-quark fusion; gluon-gluon fusion; quark-quark scattering), the several expressions we presented here will allow a direct comparison between theory and experiment. At this point a thorough detector simulation of these processes is needed to establish under which conditions, if any, they might be observed at the LHC, and what precision one might expect to obtain on bounds on the couplings $\{\alpha, \beta, \gamma\}$.

\section{References}

[1] P.M. Ferreira, O. Oliveira and R. Santos, Phys. Rev. D73 (2006) 034011.

[2] P.M. Ferreira and R. Santos, hep-ph/0601078;

[3] W. Buchmüller and D. Wyler, Nucl. Phys. B268 (1986) 621.

[4] E. Malkawi and T. Tait, Phys. Rev. D54 (1996) 5758; T. Han, K. Whisnant, B.L. Young and X. Zhang, Phys. Lett. $B 385$ (1996) 311; T. Han, M. Hosch, K. Whisnant, B.L. Young and X. Zhang, Phys. Rev. D55 (1997) 7241; K. Whisnant, J.M. Yang, B.L. Young and X. Zhang, Phys. Rev. D56 (1997) 467; M. Hosch, K. Whisnant and B.L. Young, Phys. Rev. D56 (1997) 5725; T. Han, M. Hosch, K. Whisnant, B.L. Young and X. Zhang, Phys. Rev. D58 (1998) 073008; K. Hikasa, K. Whisnant, J.M. Yang and B.L. Young, Phys. Rev. D58 (1998) 114003.

[5] F. del Àguila and J.A. Aguilar-Saavedra, Phys. Rev. D67 (2003) 014009.

[6] T. Tait and C. P. Yuan, Phys. Rev. D63, (2001) 014018; D. O. Carlson, E. Malkawi, and C. P. Yuan, Phys. Lett. B337, (1994) 145; G. L. Kane, G. A. Ladinsky, and C. P. Yuan, Phys. Rev. D45, (1992) 
124; T. G. Rizzo, Phys. Rev. D53, (1996) 6218; T. Tait and C. P. Yuan, Phys. Rev. D55, (1997) 7300; A. Datta and X. Zhang, Phys. Rev. D55, (1997) 2530; E. Boos, L. Dudko, and T. Ohl, Eur. Phys. J. C11, (1999) 473; D. Espriu and J. Manzano, Phys. Rev. D65, (2002) 073005.

[7] Gad Eilam, Mariana Frank and Ismail Turan, hep-ph/0601253; J. Guasch, W. Hollik, S. Peñaranda and J. Solà, hep-ph/0601218.

[8] J. J. Liu, C. S. Li, L. L. Yang and L. G. Jin, Phys. Rev. D72 (2005) 074018; L.L. Yang, C. S. Li, Y. Gao and J. J. Liu, hep-ph/0601180.

[9] C.T. Hill and S.J. Parke, Phys. Rev. D49 (1994) 4454; G. J. Gounaris, D. T. Papadamou, F. M. Renard, Z. Phys. C76 (1997) 333.

[10] B. Grzadkowski, J.F. Gunion and P. Krawczyk, Phys. Lett. B268 (1991) 106; G. Eilam, J.L. Hewett and A. Soni, Phys. Rev. D44 (1991) 1473; T.P. Cheng and M. Sher, Phys. Rev. D35 (1987) 3484; L.J. Hall and S. Weinberg, Phys. Rev. D48 (1993) R979.

[11] B. Grzadkowski, Z. Hioki, K. Ohkuma and J. Wudka, Nucl. Phys. B689 (2004) 108.

[12] V.M. Budnev, I.F. Ginzbirg, G.V. Meldon and V.G. Serfo, Phys. Rep. 15C (1975) 183; R. Cutler and D. Sivers, Phys. Rev. D17 (1978) 17.

[13] A. Denner and T. Sack, Nucl. Phys. B358 (1991) 46; G. Eilam, R.R. Mendel, R. Migneron and A. Soni, Phys. Rev. Lett. 66 (1991) 3105; A. Czarnecki and K. Melnikov, Nucl. Phys. 3554 (1999) 520; K.G. Chetyrkin, R. Harlander, T. Seidensticker and M. Steinhauser, Phys. Rev. D60 (1999) 114015; S.M. Oliveira, L. Brücher, R. Santos and A. Barroso, Phys. Rev. D64 (2001) 017301.

[14] J. Pumplin et al, JHEP 0207 (2002) 012.

[15] A. Belyaev and E. Boos, Phys. Rev. D63 (2001) 034012; Z. Sullivan, Phys. Rev. D70 (2004) 114012.

[16] M.E. Luke and M.J. Savage, Phys. Lett. B307 (1993) 387; D. Atwood, L. Reina and A. Soni, Phys. Rev. D55 (1997) 3156; J.M. Yang, B.L. Young and X. Zhang, Phys. Rev. D58 (1998) 055001; J. Guasch and J. Solà, Nucl. Phys. $B 562$ (1999) 3; D. Delepine and S. Khalil, Phys. Lett. B599 (2004) 62; J.J. Liu, C.S. Li, L.L. Yang and L.G. Jin, Phys. Lett. $B 599$ (2004) 92; J.A. Aguilar-Saavedra, Acta Phys. Pol. B35 (2004) 2695.

[17] S. S. Willenbrock and D. A. Dicus, Phys. Rev. D34 (1986) 155; C. P. Yuan, Phys. Rev. D41, (1990) 42; R. K. Ellis and S. Parke, Phys. Rev. D46, (1992) 3785; D. O. Carlson and C. P. Yuan, Phys. Lett. B306, (1993) 386; A. P. Heinson, A. S. Belyaev and E. E. Boos, Phys. Rev. D 56, (1997) 3114; G. Bordes and B. van Eijk, Nucl. Phys. B435, (1995) 23; T. Stelzer, Z. Sullivan and S. Willenbrock, Phys. Rev. D56 (1997) 5919; B. W. Harris, E. Laenen, L. Phaf, Z. Sullivan, and S. Weinzierl, Phys. Rev. D66, (2002) 054024; S. Cortese and R. Petronzio, Phys. Lett. B253, (1991) 494; T. Stelzer and S. Willenbrock, Phys. Lett. B357, (1995) 125; Martin C. Smith and S. Willenbrock, Phys. Rev. D54 (1996) 6696; S. Mrenna and C. P. Yuan, Phys. Lett. B416, (1998) 200; B. W. Harris, E. Laenen, L. Phaf, Z. Sullivan, and S. Weinzierl, Int. J. Mod. Phys. A16S1A, (2001) 379; T. M. P. Tait, Phys. Rev. D61, (2000) 034001; S. Moretti, Phys. Rev. D56, (1997) 7427; S. Zhu, Phys. Lett. B524 (2002) 283; Erratum-ibid. B537 (2002) 351. T. Stelzer, Z. Sullivan, and S. Willenbrock, Phys. Rev. D58, (1998) 094021. 